\title{
Understanding patients' perceptions of asthma control: a qualitative study
}

\author{
Natalie Bidad ${ }^{1}$, Neil Barnes ${ }^{2}$, Chris Griffiths ${ }^{3}$ and Rob Horne ${ }^{1}$ \\ Affiliations: ${ }^{1}$ Centre for Behavioural Medicine, UCL School of Pharmacy, London, UK. ${ }^{2}$ William Harvey \\ Institute, Barts and The London School of Medicine and Dentistry, London, UK. ${ }^{3}$ Centre for Primary Care and
} Public Health, London, UK.

Correspondence: Rob Horne, Centre for Behavioural Medicine, UCL School of Pharmacy, Mezzanine Floor, BMA House, Tavistock Square, London, WC1H 9JP, UK. E-mail: r.horneducl.ac.uk

@ERSpublications

For many asthma sufferers "control" means avoidance of deterioration which results in ineffective selfmanagement http://ow.ly/paFA30jWAez

Cite this article as: Bidad N, Barnes N, Griffiths C, et al. Understanding patients' perceptions of asthma control: a qualitative study. Eur Respir J 2018; 51: 1701346 [https://doi.org/10.1183/13993003.01346-2017].

ABSTRACT Asthma control is suboptimal for many sufferers despite the existence of effective treatments. Patients' self-management is influenced by their perceptions of asthma and its treatment. This study explored sufferers' perceptions of asthma control and their influence on self-management behaviours.

Participants $(n=42)$ recruited from primary and secondary care asthma clinics in London in the UK each underwent a qualitative interview exploring perceptions and experiences of asthma control. Purposive sampling ensured variation in disease severity, degree of asthma control, age and socioeconomic status. Grounded theory was employed in thematic analysis of transcribed interviews.

Five themes relating to perceptions of asthma control and self-management were identified: personal meaning of control, intermittent prevention, compromising control to avoid medication, pharmacological agents overemphasised in control and the role of asthma review in control. Within the first theme, some participants had an internal barometer of the level of symptoms that indicated their asthma was getting "out of control" that was set much higher than Asthma Control Test criteria.

The findings provide new insights into patients' perceptions of asthma control. Symptoms indicative of poor control were often tolerated as part of living with asthma. Identification of barriers and drivers to self-management highlight potential targets for strategies aimed at optimising asthma management. 


\section{Introduction}

Asthma is a chronic condition in which respiratory manifestations arise from systemic inflammatory processes [1]. Prevalence is high, with $\sim 8.8 \%$ of the UK population diagnosed [2]. Suboptimal management poses a public health burden and its recognition as a political priority was urged in a European Union directive [3].

Optimal management of asthma is defined in terms of preventing symptoms, minimising exacerbations or the need for rescue medication, enabling ordinary levels of physical activity and achieving near-normal lung function, while incurring minimal adverse effects from the medication $[4,5]$. These management goals are achievable outcomes irrespective of disease severity [6,7] and result in improved quality of life [8]. Despite this, asthma is poorly controlled for many $[9,10]$ and in the UK the National Review of Asthma Deaths (NRAD) highlighted avoidable contributory factors in $65 \%$ of the cases [11]. Furthermore, three European studies reported that few participants considered their asthma to be uncontrolled despite high asthma-related morbidity in a large proportion of patients [10]. This apparent divergence from the medical model of asthma as a chronic condition requiring long-term preventative medication is also evident in the literature reporting patients' beliefs about the disease [12].

Self-management, including maintaining adherence to treatment that has been appropriately prescribed and reviewed, is essential in order to achieve optimal asthma control [13]. Indeed encouragement of self-management is a key recommendation arising from the NRAD [11]. However, rather than passively following treatment advice, patients instead appraise the advice in relation to their "common sense" understanding of the condition and its treatment [14,15]. Patients perceiving incongruity between the medical model of asthma (chronic condition) and their own symptomatic experience of asthma (episodic condition) were more likely to doubt their personal need for inhaled corticosteroids (ICSs), and consequently were less adherent [15]. Similarly, a longitudinal study reported that over half the participants did not believe they had asthma when the symptoms were absent, a belief that was inversely related to favourable attitudes towards ICSs [16].

Facilitation of optimal self-management requires a thorough understanding of patients' beliefs about asthma control and the consequent influence on lifestyle behaviours. In accordance with UK Medical Research Council guidelines for developing complex interventions to effect behaviour change, this study constitutes the preparative phase in the development of an intervention to help patients identify and address poor control, through active self-management. This study employed qualitative methodology to explore patients' perceptions surrounding asthma control and self-management behaviours, to address the following research questions: 1) What are asthma patients' perceptions about asthma control and how do they make sense of the concept of asthma control? 2) How do perceptions of asthma control relate to the way in which people manage their asthma and make lifestyle choices?

\section{Methods}

Participants

This UK study was approved by the South East London Research Ethics Committee. Participants $(n=42)$ were recruited from an East London National Health Service primary care asthma clinic and The London Chest Hospital asthma outpatient clinic (table 1). Adult participants with a diagnosis of asthma requiring more than treatment with a short-acting $\beta_{2}$-agonist alone were recruited. The participant's current asthma pharmacotherapy was compared against the British Thoracic Society (BTS)/Scottish Intercollegiate Network guidelines that were in place at the time the interviews were conducted [5], and the corresponding BTS "step" was used as an indication of asthma severity. For example, BTS step 2 indicates that the participant was prescribed regular ICSs, BTS step 3 indicates that the participant was prescribed regular ICSs and a long-acting $\beta_{2}$-agonist or other add-on therapy such as theophylline, and so on up to step 5 for which participants were additionally prescribed continuous or frequent oral steroids [5]. All participants provided written informed consent. People diagnosed with chronic obstructive airways disease, any other chronic lung disease or psychotic disorders were excluded from the study, as were non-English speakers.

\section{Procedure}

Eligible patients identified by clinicians were provided a pack containing an invitation to participate in the study and validated questionnaires to assess asthma control (Asthma Control Test (ACT)) and anxiety and depression (Hospital Anxiety and Depression Scale (HADS)) [17, 18]. Returned responses were evaluated to enable purposive selection of participants for qualitative interviews (table 2). As known antecedents to asthma control [19] and adherence [20], anxiety and depression were included in the sampling criteria. In-depth individual interviews were conducted by Sarah Thum-Bonanno, a research assistant working under the supervision of author R.H., and focused on participants' perceptions and experiences of asthma 


\section{TABLE 1 Demographic variables of the 42 participants}

\begin{tabular}{|c|c|c|c|}
\hline & Primary care & Secondary care outpatients & Total \\
\hline \multicolumn{4}{|l|}{ Sex } \\
\hline Male & 8 & 11 & $17(40)$ \\
\hline Female & 17 & 8 & $25(60)$ \\
\hline \multicolumn{4}{|l|}{ Age years } \\
\hline $18-29$ & & 5 & $5(12)$ \\
\hline $30-40$ & 5 & 2 & $7(17)$ \\
\hline $41-50$ & 7 & 3 & $10(24)$ \\
\hline $51-60$ & 7 & 4 & $11(26)$ \\
\hline$>60$ & 4 & 5 & $9(21)$ \\
\hline \multicolumn{4}{|l|}{ Ethnicity } \\
\hline White & 14 & 15 & $29(69)$ \\
\hline Black Caribbean & 5 & 2 & $7(17)$ \\
\hline Black African & 1 & & $1(2)$ \\
\hline Other Black & 1 & & $1(2)$ \\
\hline Indian & 1 & & $1(2)$ \\
\hline Other Asian & 1 & & $1(2)$ \\
\hline Mixed race & & 2 & 2 (5) \\
\hline \multicolumn{4}{|l|}{ BTS step } \\
\hline 2 & 15 & & $15(36)$ \\
\hline 3 & 7 & & $7(17)$ \\
\hline 4 & 1 & 7 & 8 (19) \\
\hline 5 & & 12 & 12 (29) \\
\hline \multicolumn{4}{|c|}{ Annual household income GBP } \\
\hline $5000-11900$ & 3 & 2 & $5(12)$ \\
\hline \multicolumn{4}{|l|}{$12000-15900$} \\
\hline $16000-24900$ & 4 & 1 & $5(12)$ \\
\hline $25000-34900$ & 3 & 2 & $5(12)$ \\
\hline $35000-49900$ & 3 & & $3(7)$ \\
\hline $50000-74900$ & 3 & 1 & $4(10)$ \\
\hline \multicolumn{4}{|l|}{ Response } \\
\hline “Don't know” & & 1 & $1(2)$ \\
\hline No response given & 7 & 12 & $19(45)$ \\
\hline \multicolumn{4}{|l|}{ HADS score } \\
\hline \multicolumn{4}{|l|}{ Anxiety } \\
\hline 0-7 (normal) & 12 & 8 & $20(48)$ \\
\hline 8-10 (borderline) & 6 & 3 & $9(21)$ \\
\hline$\geqslant 11$ (clinical range) & 3 & 6 & $9(21)$ \\
\hline Not captured & 2 & 2 & $4(10)$ \\
\hline \multicolumn{4}{|l|}{ Depression } \\
\hline 0-7 (normal) & 16 & 8 & $24(57)$ \\
\hline 8-10 (borderline) & 1 & 4 & 5 (12) \\
\hline$\geqslant 11$ (clinical range) & 4 & 5 & $9(21)$ \\
\hline Not captured & 2 & 2 & $4(10)$ \\
\hline \multicolumn{4}{|l|}{ ACT score } \\
\hline$\geqslant 20$ (good control) & 7 & 3 & $10(24)$ \\
\hline$<20$ (poor control) & 16 & 14 & $30(71)$ \\
\hline Not captured & & 2 & 2 (5) \\
\hline
\end{tabular}

Data are presented as n or n (\%). BTS: British Thoracic Society; HADS: Hospital Anxiety and Depression Scale; ACT: Asthma Control Test.

control, what control means to them, how they decide whether their asthma is under or out of control, and how they manage their asthma and make lifestyle choices. The interview schedule provided a guide for the discussion, but allowed participants to raise issues pertinent to them that were then explored. Interviews were audio-recorded and transcribed verbatim.

Data analysis

Grounded theory was employed during the systematic thematic analysis of the interview transcripts. Initial codes generated from the transcripts were organised into preliminary themes that were developed and refined through discussion with the multidisciplinary research team, and cross-checked with the original 


\begin{tabular}{|c|c|c|c|c|}
\hline & BTS step $2 / 3$ & BTS step $4 / 5$ & Total & Data missing \\
\hline \multicolumn{5}{|l|}{ Sex } \\
\hline Male & 7 & 10 & 17 & \\
\hline Female & 15 & 10 & 25 & \\
\hline ACT score & & & & 2 \\
\hline Poor $(<20)$ & 15 & 15 & 30 & \\
\hline Good $(\geqslant 20)$ & 7 & 3 & 10 & \\
\hline HADS score & & & & 4 \\
\hline Borderline or in clinical range $(\geqslant 8)$ & 9 & 11 & 20 & \\
\hline Normal $(<8)$ & 11 & 7 & 18 & \\
\hline Household income & & & & 19 \\
\hline National average or below & 6 & 5 & 11 & \\
\hline Above national average & 9 & 3 & 12 & \\
\hline Age years & & & & 3 \\
\hline$\leqslant 40$ & 6 & 7 & 13 & \\
\hline$>40$ & 15 & 11 & 26 & \\
\hline
\end{tabular}

Data are presented as n. BTS: British Thoracic Society; ACT: Asthma Control Test; HADS: Hospital Anxiety and Depression Scale. " : adequate representation defined as minimum of $n=3$ per cell.

data. Selection of participants for qualitative interview continued until thematic saturation. The most specific and relevant themes were selected for inclusion in this article.

\section{Results}

Thematic analysis addressed the research questions stipulated previously, i.e. 1) what are asthma patients' perceptions about asthma control and how do they make sense of the concept of asthma control, and 2) how the perceptions of asthma control relate to the way in which people manage their asthma and make lifestyle choices? The following themes were identified from the data.

\section{Personal meaning of asthma control}

Variation in the personal meaning of asthma control was evident, specifically with regard to beliefs about the pertinent therapeutic goal. Perceptions of asthma control were related in a logical manner to perceived need for preventative medication. Participants appeared to ascribe to one of two distinct models of control: the "symptom prevention" model or the "symptom response" model.

\section{"Symptom prevention" model}

For the minority of participants, the expectation of therapy was to achieve and maintain improvement or resolution of symptoms. Occurrence of symptoms necessitating use of short-acting $\beta_{2}$-agonists was therefore an indication of poor control for these participants.

I have realised that if I don't take the preventer, then I have to take the reliever which is saying to me that my condition isn't being controlled. (Participant 11, BTS step 2, ACT score 17)

Participants ascribing to this model held the belief that prophylactic medication was necessary even in the absence of symptoms and acknowledged the importance of their asthma medication for maintaining their wellbeing.

... the drugs are much better now. I think that is the crucial factor .... If I stopped taking the drugs, I know it [asthma symptoms] will come back .... (Participant 10, BTS step 3, ACT score 18)

\section{"Symptom response" model}

The majority of participants appeared to adopt a "symptom response" model of control that centred on avoidance of deterioration rather than symptom resolution. Their therapeutic strategy was to "treat" symptoms if they exceeded an individually defined tolerance threshold. Participants appeared to have an internal barometer of the level of symptoms that indicated their asthma was "out of control". However, it was clear that for many participants the level of symptoms indicating poor control (their "tolerance threshold") was set much higher than the ACT criteria. Symptoms indicative of poor control were often tolerated as part of living with asthma. 
I get a sort of dry cough is the thing that usually makes me aware that what's probably troubling me is asthma, and then I get quite drowsy and a bit sort of not very alert and then I get a sort of tightening in the chest mainly ... and then after a while I think aha time to get the salbutamol out. (Participant 8, BTS step 2, ACT score 20)

Accordingly, these participants tended to place less significance on the importance of their asthma medications for their current state of health.

You have got to be prepared to help yourself and I personally don't think you should just rely on, oh well I have got my medication so that is it. There are always things that can go hand in hand with the medication ....don't wait until you have to ... go on the nebuliser. (Participant 18, BTS step 2, ACT score 18)

\section{Intermittent prevention}

Most participants correctly distinguished "reliever" and "preventer" medication. However, some participants' interpretation of "prevention" was discordant with prescribers' recommendations. Rather than using preventer medication regularly on a daily basis, some instead used it only to "prevent" an exacerbation when they experienced symptoms perceived to be indicative of worsening asthma. In most cases this was in response to a cold or feeling "run down". Their use of the preventer was intermittent and adherence was contextual; adherence was high when the need for "prevention" was perceived to be high and low when "prevention" was thought to be less necessary, as determined by the experience of symptoms thought to increase the risk of asthma exacerbation.

... to be honest I don't use the preventer all year because as I say it is quite seasonal and I don't want to be taking it if I am not going to be ill, but if I start feeling run down or like I am getting a cold then I start taking it and the reliever I take as and when. (Participant 17, BTS step 2, ACT score 16)

\section{Compromising asthma control to avoid medication}

Apprehensions about potential or actual adverse effects of asthma medication were frequently expressed and appeared to be a barrier to optimal control for some participants. If asthma symptoms were not intrusive they opted to avoid or reduce medication until a worsening of symptoms prompted them to perceive that treatment necessity outweighed concerns. Several subthemes emerged.

\section{Steroid phobia}

Concerns regarding the adverse effects of corticosteroids were commonly articulated, particularly by those with more severe disease. Although logical from the participant's perspective, the attribution of some adverse effects to corticosteroids was potentially exaggerated and indicates the level of anxiety for some individuals. There was a recurrent sense that corticosteroids were perceived as dangerous and harmful rather than therapeutic. Indeed, one participant used the term "steroid abuse" to refer to regular therapeutic use.

I have recently had cancer in my neck, side-effects of it [corticosteroid] and some of it's probably something to do with that, side-effects of it but I mean you don't know. But definitely long-term steroid abuse is to do with the osteoporosis. (Participant 31, BTS step 5, ACT score 5)

\section{"Relievers" benign relative to steroids}

The perception of $\beta_{2}$-agonists as being relatively benign compared with corticosteroids was evident for some. These participants opted to "control" their asthma predominantly with the short-acting $\beta_{2}$-agonists.

There was a time when it was only Ventolin but then I had to go back on Becotide again for a bit for a while, but I've taken it on and off, even though I was asked to take it on a regular basis, I was a bit naughty but I thought I can cope without it. I don't regret it. (Participant 15, BTS step 2, ACT score 15)

\section{Conservation of efficacy}

Beliefs and concerns regarding refractoriness to bronchodilators with excessive or prolonged use and reaching a ceiling therapeutic effect were expressed by participants who harboured a desire to conserve efficacy. These understandings impacted upon adherence to medication. 
... I like to think that I am taking the minimum amount of medication so there is headroom in there for when there is an asthma attack for a drug to have more time to work .... (Participant 39, BTS step 4, ACT score 13)

Reluctance to be dependent on medication

Some participants indicated a reluctance to be reliant on asthma medications and so instead attempted to cope without them.

... I usually just bring a bottle of water around with me so that can ... stop that [asthma symptoms] rather than to keep taking the pump to try and keep on top of it, because sometimes the more you rely on the pump the more you can end up taking them. (Participant 23, BTS step 2, ACT score 21)

\section{Dynamic relationship between ICS concerns and adherence}

There was evidence that the relationship between medication concerns and control (and therefore adherence) was a dynamic process, fluctuating with acute worsening of symptoms, disease progression or time.

... when I was younger I was much more resistant to using inhalers... I wanted to get over it with my own resources ... so I've changed my view a bit ... I think well it's not going to harm me that much taking an inhaler. It probably does me more harm struggling to breathe .... (Participant 9, BTS step 2, ACT score 18)

\section{Asthma control and asthma review}

Perceptions of asthma management and interactions with healthcare professionals tended to be more positive for secondary care participants. A negative perception of asthma review appointments was commonly articulated by primary care participants, some describing it as a waste of time or a bureaucratic step to obtaining a prescription. Poor understanding of peak flow, why it was measured and how to interpret the results was also evident. Some participants did not perceive the review clinic as an essential aid to asthma control, but rather as an additional burden.

I am quite pragmatic. I have to have a review in order to get the medicine because you can't have an open ended prescription. So that is what the review is about, but it is not really about asthma management or the course of a condition .... (Participant 11, BTS step 2, ACT score 17)

This perception was compounded by the highly variable and sometimes conflicting advice seen to be provided by generalist healthcare professionals.

I've had conflicting advice about peak flow, I think I had one young doctor and he said my peak flow was ... not very good at all which I found a bit alarming ... and then I saw the nurse and she said oh no that's alright .... (Participant 9, BTS step 2, ACT score 18)

\section{Asthma control overemphasises medicines}

Nearly all participants began by talking about their medicines when first invited to discuss their asthma diagnosis and control. Medication was therefore perceived by the participants as a fundamental aspect of their condition and central to its control. However, despite acknowledging the centrality of medicines to asthma control, many participants also expressed the apparently conflicting belief that healthcare professionals focused asthma management too heavily towards pharmacological treatments.

Medicine, medicine, medicine, peak flow, medicine, no mention of management, no mention of behaviour, no mention of breathing exercises, no mention of alternatives, you are an asthmatic take your inhaler. That is what you get from doctors as an asthmatic in the UK, that is all I have ever got. (Participant 24, BTS step 2, ACT score 22)

Many expressed a desire for a more holistic approach with advice on lifestyle incorporated. Nontraditional approaches to controlling asthma symptoms played an important role in asthma control for many participants. For some, alternative methods were perceived as preferable to conventional treatment, whereas for others complimentary techniques were used in combination with prescribed therapy and benefit was perceived to be additive. 
I work with a number of natural healers, a naturopath and an acupuncturist who help me control it [asthma] ... all the drugs are a last resort for me. (Participant 8, BTS step 2, ACT score 20)

\section{Discussion}

This study provides new insight into patients' perspectives of asthma control and extends existing understanding of why some people with asthma do not engage with optimal treatment. This study found participants to perceive medication as central to asthma control, although many felt there to be an overemphasis on pharmacological management. Participants conceptualised asthma control according to one of two distinct models: the "symptom prevention" model, which concurs with the medical view of asthma as a chronic condition requiring prophylactic medication, or the "symptom response" model, which centres on the avoidance of deterioration rather than achieving improvement in functioning. For the latter, the participants appeared to have an internal barometer of the level of symptoms indicating their asthma was "out of control" that was set much higher than ACT criteria. Participants" "model" of asthma control appeared to influence their perceived need for preventer medication and hence their adherence in a logically consistent way. Some extrapolated the "reliever" paradigm to the use of the "preventers", such that the latter were being used on an as-required basis to prevent deterioration during acute worsening of symptoms. Many participants perceived there to be high costs associated with medications, e.g. loss of efficacy, dependence and side-effects, the latter particularly for corticosteroids.

Consistent with previous research, some participants were found to avoid medication to the detriment of their asthma control [21]. Adherence was related to beliefs about treatment and in particular how personal need for the preventer inhaler is judged relative to concerns about potential adverse consequences of adhering to it $[15,22,23]$. The notion that necessity beliefs and concerns about medicines can influence adherence in chronic conditions (including asthma) is well supported by published literature [24]. Furthermore, the apparent discrepancy between patients' beliefs and the medical understanding of asthma treatment is consistent with previous qualitative work [25]. In the current study, many of the medicine-taking strategies reported by the interviewees appeared to be suboptimal in terms of medical recommendations for maintaining asthma control. However, they seemed to represent "common sense" approaches to managing asthma when viewed from the participants' perspective. In particular, participants often downplayed the importance of daily preventer treatments if they felt their asthma was not bothersome at the time. In this respect, the findings suggest an incongruence between the medical perspective, emphasising proactive control through prevention of exacerbations, and the patient's perspective, where to some extent symptoms are regarded as part and parcel of having asthma, rather than a sign that their asthma is poorly controlled. These findings are consistent with previous studies showing that nonadherence often results when the patient perceives inconsistency between their understandings of their illness and the treatment $[15,16,26]$.

Using qualitative methodology, we sought to gain in-depth understanding of asthma control from a small sample and consequently the views expressed may have limited generalisability. However, the sample was heterogeneous and our findings are consistent with and extend upon previous research. This study did not attempt to explore how peoples' perceptions of asthma control change over time or with disease progression, or how "tolerance thresholds" are developed, and further research is needed to address these important aspects. A quantitative analysis to investigate the proportion of patients with asthma that adopt each "model" of control and how this relates to severity of asthma would be an interesting and important extension of this work. In addition, a quantitative study to investigate the relationship between beliefs about asthma control and self-management behaviour (e.g. adherence to prophylactic medication) is warranted in light of the findings.

This study represents the critical first step informing the development of a targeted intervention that will ultimately help reduce the economic burden of asthma and improve the lives of asthma sufferers. The "cognitive disconnect" evident between the medical and patient understanding of asthma control and pharmacotherapy, and the influence of underlying perceptions on self-management, serves to underline that simply providing accurate information about asthma and its management is not enough to ensure that patients take their treatment according to medical advice [27]. Instead, illness and treatment beliefs must be first elicited by healthcare professionals and then strategies implemented to address any misconceptions about treatment necessity and/or concerns. Adherence-related beliefs of asthma and ICSs can be elicited using validated questionnaires (e.g. the Illness Perceptions Questionnaire [28] measures how people understand their illness, and the Beliefs about Medicines Questionnaire [29] quantifies perceptions around medication necessity and concerns). The study authors are using the current findings to inform the modification of these validated questionnaires to develop a practical tool for use in clinical settings. This tool will enable quick identification of underlying misconceptions about asthma control and medication, and enable targeted messages to correct specific misconceptions or adherence-related 
concerns. The feasibility of such an approach has been demonstrated in previous work, in which a text message intervention demonstrated favourable changes in illness and medication beliefs, and improved adherence in patients with asthma [30].

In this study the unfavourable opinion of asthma review clinics expressed by some participants may be a cause for concern, given the inclusion of asthma reviews in the general practitioner Quality and Outcomes Framework in the UK. Insufficient information on the "what to" and "how to" communicate with asthmatic patients has been identified previously for explaining the poor uptake of self-management plans and guideline use by primary care professionals [31]. The timing of discussions around adherence also seems to be critical factor [32]. Furthermore, effective collaboration between healthcare professionals and patients with asthma was found to be hampered by failure of the healthcare professionals to elicit, and the patient to disclose, health beliefs inconsistent with Western medicine or those that are simply inaccurate [33]. This may be because patients believe that expressing doubts or concerns about treatment may lead the clinician to doubt them. For this reason a no-blame approach is needed as the first step in facilitating an honest and open discussion about asthma control and optimal self-management [34].

\section{Conclusions}

This study has identified potentially important barriers and drivers to self-management behaviour aimed at maintaining and improving control (e.g. adherence to preventer medication). The understandings gained are the critical first step for informing the ongoing development of an intervention targeted to help patients develop realistic expectations of asthma control and more effective self-management practices. A successful intervention will therefore be valuable for patients and healthcare professionals as both would derive personal benefit from mutually satisfactory consultations and optimised care. Furthermore, interventions aimed at optimising asthma control will assist in the achievement of the European Union directive [3] and therefore the insights gained from this study have implications at the societal level.

Acknowledgements: The authors would like to thank Sarah Thum-Bonanno, who conducted the semistructured interviews, for her valuable contribution to this study. She was working at the Centre for Behavioural Medicine, UCL School of Pharmacy (London, UK) under the supervision of R. Horne at the time she conducted the interviews.

Conflict of interest: N. Barnes is employed by and holds shares in GSK. R. Horne reports personal fees for speaker engagements from AbbVie, Amgen, Biogen, Idec, Gilead Sciences, GSK, Janssen, Pfizer, Roche, Shire Pharmaceuticals, MSD, Astellas, AstraZeneca, DRSU, Novartis and Universitatsklinikum Hamburg-Eppendorf, and is a funder and shareholder of Spoonful of Sugar Ltd, during the conduct of the study. R. Horne was supported by the National Institute for Health Research (NIHR) Collaboration for Leadership in Applied Health Research and Care (CLAHRC) North Thames at Bart's Health NHS Trust. The views expressed are those of the author(s) and not necessarily those of the NHS, the NIHR or the Dept of Health.

Support statement. This study was funded by Asthma UK (grant 09/026). Funding information for this article has been deposited with the Crossref Funder Registry.

\section{References}

1 Leif B. Time for a paradigm shift in asthma treatment: from relieving bronchospasm to controlling systemic inflammation. J Allergy Clin Immunol 2007; 120: 1269-1275.

2 Asthma UK. Key facts and statistics. 2017. www.asthma.org.uk/about/media/facts-and-statistics Date last accessed: May 12, 2018.

3 Holgate S, Bisgaard H, Bjermer L, et al. The Brussels Declaration: the need for change in asthma management. Eur Respir J 2008; 32: 1433-1442.

4 Global Initiative for Asthma. Global Strategy for Asthma Management and Prevention. 2017. http://ginasthma.org/ 2017-gina-report-global-strategy-for-asthma-management-and-prevention Date last accessed: May 12, 2018.

5 British Thoracic Society/Scottish Intercollegiate Guidelines Network. British Guideline on the Management of Asthma. 2014. www.brit-thoracic.org.uk/document-library/clinical-information/asthma/btssign-asthma-guideline2014 Date last accessed: May 12, 2018.

6 Bateman ED, Bousquet J, Braunstein GL. Is overall asthma control being achieved? A hypothesis-generating study. Eur Respir J 2001; 17: 589-595.

7 Bateman ED, Boushey HA, Bousquet J, et al. Can guideline-defined asthma control be achieved? The Gaining Optimal Asthma ControL study. Am J Respir Crit Care Med 2004; 170: 836-844.

8 Bateman ED, Frith LF, Braunstein GL. Achieving guideline-based asthma control: does the patient benefit? Eur Respir J 2002; 20: 588-595.

9 Partridge MR, van der Molen T, Myrseth SE, et al. Attitudes and actions of asthma patients on regular maintenance therapy: the INSPIRE study. BMC Pulm Med 2006; 6: 13.

10 Holgate ST, Price D, Valovirta E. Asthma out of control? A structured review of recent patient surveys. BMC Pulm Med 2006; 6: Suppl. 1, S2.

11 Royal College of Physicians. Why Asthma Still Kills: The National Review of Asthma Deaths (NRAD). Confidential Enquiry Report. London, Royal College of Physicians, 2014.

12 Ring N, Jepson R, Hoskins G, et al. Understanding what helps or hinders asthma action plan use: a systematic review and synthesis of the qualitative literature. Patient Educ Couns 2011; 85: e131-e143. 
13 Horne R, Price D, Cleland J, et al. Can asthma control be improved by understanding the patient's perspective? BMC Pulm Med 2007; 7: 8.

14 Horne R. Compliance, adherence, and concordance: implications for asthma treatment. Chest 2006; 130: 1 Suppl., 65S-72S.

15 Horne R, Weinman J. Self-regulation and self-management in asthma: exploring the role of illness perceptions and treatment beliefs in explaining non-adherence to preventer medication. Psychol Health 2002; 17: 17-32.

16 Halm EA, Mora P, Leventhal H. No symptoms, no asthma: the acute episodic disease belief is associated with poor self-management among inner-city adults with persistent asthma. Chest 2006; 129: 573-580.

17 Nathan RA, Sorkness CA, Kosinski M, et al. Development of the asthma control test: a survey for assessing asthma control. J Allergy Clin Immunol 2004; 113: 59-65.

18 Zigmond AS, Snaith RP. The Hospital Anxiety and Depression Scale. Acta Psychiatr Scand 1983; 67: 361-370.

19 Strine TW, Mokdad AH, Balluz LS, et al. Impact of depression and anxiety on quality of life, health behaviours, and asthma control among adults in the United States with asthma, 2006. J Asthma 2008; 45: 123-133.

20 DiMatteo MR, Lepper HS, Croghan TW. Depression is a risk factor for noncompliance with medical treatment. Arch Intern Med 2000; 160: 2101-2107.

21 Steven K, Marsden W, Neville RG, et al. Do the British Guidelines for Asthma Management facilitate concordance? Health Expect 2004; 7: 74-84.

22 Clatworthy J, Price D, Ryan D, et al. The value of self-report assessment of adherence, rhinitis and smoking in relation to asthma control. Prim Care Respir J 2009; 18: 300-305.

23 Menckeberg TT, Bouvy ML, Bracke M, et al. Beliefs about medicines predict refill adherence to inhaled corticosteroids. J Psychosom Res 2008; 64: 47-54.

24 Horne R, Chapman S, Parham R, et al. Understanding patients' adherence-related beliefs about medicines prescribed for long term conditions: a meta-analytic review of the necessity-concerns framework. PLoS One 2013; 8: e80633.

25 Peláez S, Bacon SL, Aulls MW, et al. Similarities and differences between asthma healthcare professional and patient views regarding medication adherence. Can Respir J 2014; 21: 221-226.

26 Leventhal H, Brissette I, Leventhal E, eds. The Common-Sense Model of Self-Regulation of Health and Illness. London, Routledge, 2003.

27 Horne R. Decisions about medicines: scientific evidence in context. Academy of Medical Sciences. 2017. https:// acmedsci.ac.uk/file-download/80849939 Date last accessed: January 10, 2018

28 Moss-Morris R, Weinman J, Petrie KJ, et al. The revised Illness Perception Questionnaire (IPQ-R). Psychol Health 2002; 17: $1-16$

29 Horne R, Weinman J, Hankins M. The beliefs about medicines questionnaire: the development and evaluation of a new method for assessing the cognitive representation of medication. Psychol Health 1999; 14: 1-24.

30 Petrie KJ, Perry K, Broadbent E, et al. A text message programme designed to modify patients' illness and treatment beliefs improves self-reported adherence to asthma preventer medication. Br J Health Psychol 2012; 17: $74-84$.

31 Moffat M, Cleland J, van der Molen T, et al. Poor communication may impair optimal asthma care: a qualitative study. Fam Pract 2007; 24: 65-70.

32 Peláez S, Bacon SL, Lacoste G, et al. How can adherence to asthma medication be enhanced? Triangulation of key asthma stakeholders' perspectives. J Asthma 2016; 53: 1076-1084.

33 Newcomb PA, McGrath KW, Covington JK, et al. Barriers to patient-clinician collaboration in asthma management: the patient experience. J Asthma 2010; 47: 192-197.

34 Horne R. Improving adherence with asthma therapies. In: Polosa R, Papale G, Holgate ST, eds. Advances in Asthma Management. London, Future Medicine, 2012; pp. 132-142. 